\title{
Troubles métaboliques associés aux antipsychotiques atypiques : consensus belge sur la conduite à tenir
}

\author{
A. DE NAYER( ${ }^{(1)}$, M. DE HERT (2), A. SCHEEN ${ }^{(2)}$, L. VAN GAAL (4), J. PEUSKENS (2)
}

\section{Conference report : Belgian consensus on metabolic problems associated with atypical antpsychotics}

Summary. Background - The current literature supports that schizophrenia (and bipolar disorders) appear to be associated with a higher prevalence of type 2 diabetes. Because of the silent nature of diabetes mellitus, and the fact that schizophrenic patients are not screened comprehensively for the disease, the true prevalence of hyperglycemia and diabetes may be substantially underestimated (4-8). Notably, it has been suggested that schizophrenia as such carries an increased risk, as certain characteristics of schizophrenic patients such as unhealthy life style promote the diabetes risk. Literature findings - This risk may be increased by antipsychotic drug treatment, as was already suggested for firstgeneration antipsychotics (FGA) (21). The amount of literature on the association of SGA and metabolic disorders is much larger however, although well-controlled prospective data are sparse. Reports comprise abnormal glucose regulation, exacerbation of existing type 1 and 2 diabetes, new-onset pseudo-type 1 or type 2 diabetes, diabetic ketoacidosis, coma and death. In large-scale studies (mostly retrospective), reviews and meta-analyses, the association was not found for all drugs (31-34). New data - According to recent reviews, the risk of developing diabetes was highest for clozapine and olanzapine, followed by quétiapine and risperidone. The hierarchy of liability of weight gain, or differential effects on insulin resistance was also in the described order. Apart from disturbances in glucose metabolism, further frequent metabolic abnormalities in schizophrenic patients on SGA include features of the metabolic syndrom (1-6). Antipsychotics such as clozapine and olanzapine have also been associated with hypertriglyceridemia (6), while agents such as haloperidol, risperidone and ziprasidone were associated with reductions in plasma triglycerides (9). Amisulpride, aripiprazole and ziprasidone seem to carry the lowest risk for weight gain, diabetes and effects on insulin resistance. Conclusion As a consequence, there is a shift in attention toward physical health monitoring in patients with mental health disorders (21-24). The APA and ADA as well a British working group (29) have recently published the findings on SGA and metabolic abnormalities in a joint statement (table I) (6).

Key words : Diabetus mellitus ; Metabolic syndrom ; Prevention ; Schizophrenia ; Second generation atypics.

Résumé. Des psychiatres, des diabétologues et des pharmaciens des cinq centres universitaires et d'hôpitaux belges, impliqués dans le traitement de la schizophrénie, se sont réunis en vue d'effectuer une revue critique des informations les plus récentes traitant du risque d'apparition et de développement des troubles métaboliques induits par les antipsychotiques de seconde génération. Les membres du consensus ont formulé des recommandations de bonne pratique clinique psychiatrique. Certains SGA sont associés à un risque plus élevé de troubles métaboliques; dès lors avant d'entreprendre un traitement, tous les facteurs de risques métaboliques doivent être considérés. Lors du choix, il y a lieu de soigneusement soupeser à efficacité égale, à la fois le risque d'induction d'un trouble métabolique et les risques de facteurs métaboliques intrin-

(1) Hôpital Sainte-Thérèse, B-6061 Montignies-sur-Sambres.

(2) Clinique Sint-Jozef, Kortenberg.

(3) Division of diabetes, Nutrition and metabolic disorders, department of medicine, CHU Sart Tilman, Liège.

(4) Department of diabetology, metabolism and nutrition, Antwerp.

Travail reçu le $1^{\mathrm{er}}$ avril 2005 et accepté le 12 octobre 2005.

Tirés à part : De Nayer (à l'adresse ci-dessus). 
sèques au patient. Il y a lieu de privilégier le SGA aux facteurs de risques métaboliques moindres. Les stades précoces du diabète, de l'obésité, des dyslipémies doivent être décelés durant les premiers mois du traitement et si nécessaire traités. Un arrêt du SGA impliqué ainsi qu'un switch vers un SGA à risque moindre font partie de nos recommandations. Le psychiatre doit informer et créer une collaboration avec son patient, sa famille et les autres soignants.

Mots clés: Antipsychotiques de seconde génération; Diabète; Prévention ; Schizophrénie ; Syndrome métabolique.

\section{INTRODUCTION}

La littérature actuelle rapporte un taux de prévalence élevé de diabète et de troubles métaboliques auprès des patients souffrant de schizophrénie et de troubles bipolaires.

L'évolution torpide et silencieuse du diabète ainsi que sa non-détection systématique laissent à penser que la prévalence d'hyperglycémie et du diabète est probablement plus élevée encore que celles énoncées dans la littérature (25-32).

Le taux de risque de diabète plus élevé de cette population s'inscrit en conséquence du mode de vie inapproprié et de la prévalence d'un surpoids. Ce dernier et l'obésité sont associés à l'insulino-résistance et aux troubles métaboliques. Cependant la seule présence d'une obésité intra-abdominale constitue un facteur de risque plus spécifique que l'élévation du BMI. Dès lors, la simple mesure de la taille est recommandée pour déceler la composante pondérale du trouble métabolique.

L'obésité n'est cependant pas la seule étiologie. En effet, certains patients peuvent développer plusieurs facteurs de risque métabolique lors d'une élévation marginale du tour de taille : pour les hommes, de 94 à 102 et pour les femmes, de 80 à 88 . De tels patients peuvent être tributaires d'une forte contribution génétique à l'insulinorésistance. Ils doivent bénéficier de changements d'habitudes de vie, similaires aux patients se situant dans la catégorie de tour de taille plus élevé.

L'ADA (American Diabetes Association) a récemment établi la limite à $100 \mathrm{mg} / \mathrm{dl}$. Ce point limite sera d'application pour identifier la frontière inférieure de définition d'une glycémie élevée en tant que critère pour un syndrome métabolique.

L'hypothèse de risques héréditaires communs aux troubles psychotiques et métaboliques peut être envisagée.

Ce risque se voit encore augmenté par certains antipsychotiques. Ces effets secondaires déjà décrits pour les antipsychotiques de la première génération se voient toutefois amplifiés par ceux de la seconde. II est vrai que les publications concernant ces derniers sont bien plus nombreuses et plus étoffées.

II nous manque toutefois des études prospectives validées. Seuls, à notre connaissance, Howes et al. (15) ont publié une étude de ce type concernant la clozapine.
De nombreux cas rapportés dénoncent l'apparition de troubles métaboliques, d'exacerbation de troubles diabétiques existants de type I et II, de nouveaux cas « pseudotype I et II » (non liés à un gain pondéral), de cas d'acidocétose et même de comas et de décès.

D'amples études, la plupart rétrospectives, ainsi que des méta-analyses ont été effectuées. Elles ne font pas montre de risques similaires pour tous les antipsychotiques atypiques (2-3).

Deux consensus, l'un britannique, l'autre américain, ont été publiés à ce jour.

Parmi les nombreuses études rétrospectives, citons Koller et al. qui ont compilé des données du Food and Drug Administration Medwatch System, afin de relever les cas d'hyperglycémie, de diabète associés à la clozapine (19), l'olanzapine (18), la rispéridone (17) et la quétiapine (20). En vue de situer l'importance de la problématique, 237 cas sous olanzapine ont développé un diabète ou une hyperglycémie entre les années 1994 et 2001. Parmi ceux-ci, 188 nouveaux cas, 40 cas d'exacerbation de diabète pré-existant et 5 non classifiés. Les diabètes sont apparus dans un délai de 2 jours à 45 mois après l'initiation du traitement, $73 \%$ dans un intervalle de temps inférieur à 6 mois. Aucune corrélation n'a été trouvée entre posologie et glycémie ou entre posologie et délai d'apparition ; 80 patients souffraient d'une acidose métabolique ou d'une cétose. Des valeurs glycémiques supérieures ou égales à $1000 \mathrm{mg} / \mathrm{dL}$ ont été détectées chez $41 \%$ des patients, 15 patients sont décédés. Lors de l'arrêt de l'olanzapine ou lors d'une diminution posologique, $78 \%$ des cas ont connu une amélioration glycémique.

Plusieurs études récentes indépendantes attestent d'un risque de diabète accru induit par la clozapine (19) et l'olanzapine (18), suivies par la quétiapine (20) et la rispéridone (17). La prise de poids et la résistance à l'insuline connaissent la même hiérarchie (13).

En sus du trouble du métabolisme glucosé, il existe une corrélation entre les SGA (Second Generation Antipsychotics) et l'apparition d'un syndrome métabolique (1720).

La clozapine et l'olanzapine sont également associées à l'apparition d'hypertriglycéridémie.

L'amisulpride, l'aripiprazole et la ziprasidone semblent présenter le risque le moins élevé de prise de poids, de diabète, de troubles lipidiques et d'augmentation de la résistance à l'insuline.

L'étude de Howes et al. (15) précitée montre l'apparition d'anomalies glycémiques chez $55 \%$ des patients, dans les trois mois qui suivent l'initiation du traitement par la clozapine, non associées à un gain de poids.

Les mécanismes sous-jacents peuvent être la conséquence d'un effet génétique direct, d'une diminution de la sensibilité à l'insuline, indépendante de la médication, ou d'une augmentation de l'insulino-résistance, dépendante des SGA, d'une production diminuée d'insuline ou d'un effet des SGA sur les récepteurs 5HT2C. 
Au vu de ces données, on se doit de porter une attention croissante à la condition physique des patients présentant des troubles psychiatriques graves (12). L'APA et l'ADA (American Diabetic Association) (6) ont publié de concert leurs recommandations (tableau $\mathrm{I}$ ). Ces recommandations sont toutefois, à nos yeux, trop peu « sensibles »: basées uniquement sur une glycémie à jeun, la moitié des patients qui souffrent d'une intolérance glucidique échappe à ce diagnostic (7).

TABLEAU I. - SGA et troubles métaboliques.

\begin{tabular}{lccc}
\hline \multicolumn{1}{c}{ Médication } & $\begin{array}{c}\text { Prise } \\
\text { de poids }\end{array}$ & $\begin{array}{c}\text { Risques } \\
\text { de diabète }\end{array}$ & $\begin{array}{c}\text { Risques de } \\
\text { dyslipidémie }\end{array}$ \\
\hline Clozapine & +++ & + & + \\
Olanzapine & +++ & + & + \\
Rispéridone & ++ & $?$ & $?$ \\
Quétiapine & $+/-$ & $?$ & $?$ \\
Ziprasidone & $+/-$ & NP & NP \\
Aripiprazole & $+/-$ & NP & NP \\
Amisulpride & $+/-$ & NP & NP \\
\hline
\end{tabular}

Tableau adapté à partir du consensus de l'APA et de l'ADA. + : risque accru ; ? : données variables ; NP : pas de cas publié.

En outre, la fréquence des contrôles de détection telle que recommandée par ces associations, tant pour la glycémie que pour les lipides, est par trop espacée.

Lors d'un changement d'antipsychotique, ces troubles du métabolisme glucidique sont réversibles, du moins en cas de détection précoce. Cette constatation clinique entraîne à nos yeux une fréquence de recherche de détection plus élevée, lors des six premiers mois d'application d'un nouveau traitement.

Selon nous, les troubles lipidiques exigent également un suivi plus fréquent que celui préconisé par l'APA et l'ADA.

\section{COMMENT GÉRER LE RISQUE MÉTABOLIQUE ?}

Les patients schizophrènes, même non traités, présentent un risque plus élevé d'apparition de troubles métaboliques par comparaison à une population témoin. De façon notable, ils sont prédisposés à développer un diabète (2532). Ce fait implique une approche particulière. La visite initiale, avant la prescription d'un atypique revêt une importance particulière. Le patient possédant des facteurs de risque de complication métabolique doit être identifié dès le départ. Une anamnèse personnelle et familiale ayant trait aux troubles métaboliques doit être établie de façon détaillée par le psychiatre.

L'utilisation de paramètres cliniques (tableau II) présente un intérêt certain en vue d'identifier les patients à risque. II y aurait également lieu de procéder à des analyses de laboratoire. La mesure du glucose à jeun est un minimum requis.
TABLEAU II. - Identification clinique du syndrome métabolique selon l'ATP III.

\begin{tabular}{lc}
\hline \multicolumn{1}{c}{ Facteur de risque } & Norme \\
\hline $\begin{array}{l}\text { Obésité abdominale, tour de taille } \\
\text { homme }\end{array}$ & $>102 \mathrm{~cm}$ \\
femme & $>88 \mathrm{~cm}$ \\
Triglycérides & $150 \mathrm{mg} / \mathrm{dl}$ \\
$\mathrm{HDL}$ & \\
$\quad$ homme & $<40 \mathrm{mg} / \mathrm{dl}$ \\
femme & $<50 \mathrm{mg} / \mathrm{dl}$ \\
Tension artérielle & $130 / 85 \mathrm{mmHg}$ \\
Glycémie à jeun & $110 \mathrm{mg} / \mathrm{dl}$ \\
\hline
\end{tabular}

Source : National Panel on Detection, Evaluation and Treatment of High Blood Cholesterol in Adults (NCEP), Adult Treatment Program III.

La détection peut se voir affinée par la mesure de l'insuline à jeun et sa sensibilité suivant le modèle homéostatique (Homa-IR). Le calcul de la résistance de l'insuline (Homa-IR) à partir du glucose et de l'insuline à jeun a été suggéré comme ayant une valeur prédictive du diabète dans une étude belge prospective (7).

Cependant, les niveaux d'insuline ne sont pas standardisés et la mesure de celle-ci ne fait pas encore partie du protocole de détection standard. L'hyperglycémie provoquée per os (HYPO) (Oral Glucose Tolerance Test, OGTT) reste la mesure de référence pour les populations identifiées par l'anamnèse comme étant à risque. Cette mesure permet de mettre en exergue $50 \%$ de cas supplémentaires susceptibles d'évoquer un diabète par rapport à la seule glycémie à jeun. Dans la pratique quotidienne, une glycémie à jeun est plus aisée (1), cependant son manque de sensibilité peut occulter la détection d'un ensemble de patients diabétiques (7).

La HBA1C, l'hémoglobine glycosylée permet la détection de la moyenne glycémique des six à huit semaines précédant la date de la prise de sang, elle ne convient pas toutefois à la détection des patients souffrant de diabète.

Soulignons que les prédicteurs des cas les plus sévères, à savoir l'acido-acétose ne sont pas connus.

Lors du choix d'un SGA, à efficacité équivalente, le risque métabolique doit être pris en compte : les antipsychotiques, tels que l'amisulpride, l'aripiprazole et la ziprazidone possédant une faible propension à une augmentation pondérale et à l'induction du diabète seront préférés (tableau I).

Lors du choix d'un antipsychotique à hauts risques d'effets métaboliques, un suivi plus intense doit être exercé et le médecin généraliste ainsi que l'interniste devraient en être informés.

L'arrêt tabagique doit être recommandé à tous les patients, de par l'existence d'une littérature convaincante établissant un lien causal entre le tabagisme et les risques qu'il engendre. Fumer entrave le transport du glucose. Certes, un gain pondéral peut apparaître lors de l'arrêt du 
tabac, il est donc recommandé de donner des conseils quant au mode de cessation tabagique. Il est bien évidemment préférable de prévenir un gain de poids que devoir le traiter ultérieurement.

\section{QUEL SUIVI POUR LES PATIENTS SOUS ANTIPSYCHOTIQUES ?}

On recommandera aux patients de mesurer et noter leur poids et leur tour de taille. Une évaluation régulière de la glycémie et des lipides sera planifiée. Le suivi du poids et de la tension artérielle doit être recommandé.

\section{RECOMMANDATIONS LORS DE L'APPARITION D'UN TROUBLE GLYCÉMIQUE OU D'UN DIABÈTE SOUS ATYPIQUE}

Lors d'une glycémie supérieure ou égale à $100 \mathrm{mg} / \mathrm{dL}$, une confirmation de cette mesure par un re-test sera demandée dans les jours qui suivent ; les facteurs de risque seront ensuite évalués et la fréquence du suivi augmentée.

La consultation d'un généraliste ou d'un diabétologue sera requise. On procédera aux calculs du Homa-IR et à la mesure des OGTT. En fonction du résultat, une prescription d'antipsychotique non associé à un gain significatif de poids ou de diabète (tableau l) sera effectuée.

De plus, il est hautement souhaitable de procéder à un changement des habitudes de vie, de suivre des conseils diététiques, et l'administration de metphormine pour prévenir la progression du diabète peut être prise en considération.

Une glycémie à jeun dépassant ou égalant $126 \mathrm{mg} / \mathrm{dL}$ lors d'une prise de sang subséquente laisse signifier un diagnostic de diabète, le patient sera dès lors switché vers un antipsychotique non associé à un gain pondéral ou un diabète (tableau I).

Si un switch n'est guère possible ou s'il est difficile (exemple : patient sous clozapine), le diabète doit être traité avec des mesures non médicamenteuses telles qu'un changement du style de vie et des mesures diététiques, ou avec un traitement médicamenteux tel qu'un antidiabétique oral ou de l'insuline. Nous recommandons la consultation d'un médecin interniste ou d'un généraliste.

Lors de l'établissement du diagnostic du diabète, rappelons la réversibilité possible de celui-ci lors de l'arrêt rapide de l'antipsychotique.

\section{GESTION D'UNE DYSLIPIDÉMIE SOUS ATYPIQUE}

Lors du développement d'une dyslipémie sous atypique, après test de confirmation, des mesures diététiques seront prises. Si celles-ci s'avèrent non satisfaisantes, un switch vers un antipsychotique avec risque métabolique plus faible est recommandé (tableau I).
Si le changement de médication est contre-indiqué, on demandera la consultation d'un médecin généraliste ou d'un spécialiste qui initiera le traitement en conséquence, par exemple un hypocholestérolémiant.

\section{GESTION D'UNE OBÉSITÉ SOUS ANTIPSYCHOTIQUE}

II tombe sous le sens que prévenir la prise de poids est de loin préférable... Lequel poids sera prévenu par le choix d'un antipsychotique sans répercussion pondérale lors de la consultation de patients au poids trop élevé. On recommandera à ces patients un changement de style de vie et l'utilisation de mesures diététiques requises lors de l'installation et durant la thérapie antipsychotique. Une diminution des graisses, du contenu énergétique de l'apport alimentaire et l'augmentation de la prise de fibres seront de mise. Fruits et légumes seront privilégiés. Les hydrates de carbone complexes, l'évitement des boissons sucrées et procéder à des exercices physiques d'au moins dix à quinze minutes par jour (10), idéalement trente minutes (16), seraient souhaitables.

Lors d'un gain de poids significatif, à savoir plus de $7 \%$ du poids corporel, l'incidence métabolique s'accroissant, un changement vers une médication non associée à un gain de poids devrait être considéré. Au cas où ces pratiques s'avèrent insuffisantes, la consultation d'un médecin généraliste, d'un interniste et/ou d'un diététicien s'impose pour y porter remède. Les mesures médicamenteuses telles que la prise d'Orlistat (absence d'action stimulante centrale ; procède par inhibition des lipases gastro-intestinales et diminue ainsi la résorption des graisses du bol alimentaire) sont onéreuses ; elles peuvent, en outre, provoquer la malabsorption d'un antipsychotique. La sibutramine a été suspectée d'induire des symptômes psychotiques et est donc contre-indiquée aux patients présentant des troubles psychiatriques.

\section{RECOMMANDATIONS QUANT À UNE COLLABORATION ENTRE UN PSYCHIATRE ET UN DIABÉTOLOGUE}

Selon les présentes recommandations, le psychiatre initie la détection et les mesures de suivi. Au cas où les différents examens de laboratoire font montre d'une perturbation du métabolisme glucosé, de l'apparition d'un diabète, d'une dyslipidémie ou d'une hypertension, le suivi de ces maladies physiques doit être au préalable entrepris par un médecin traitant ou un interniste, voire un diabétologue. Une communication étroite doit être maintenue entre le psychiatre et le médecin traitant, tout spécialement lors d'un changement ou d'une confirmation d'une médication antipsychotique.

Le but premier de la thérapie est d'arriver au meilleur contrôle de la maladie mentale, cependant les troubles métaboliques doivent désormais également entrer en ligne de compte. 


\section{RECOMMANDATIONS AUX PATIENTS SOUFFRANT D'AUTRES TROUBLES MENTAUX QUE LA SCHIZOPHRÉNIE, TRAITÉS PAR LES ATYPIQUES}

Les atypiques sont utilisés dans un spectre d'indications incluant les troubles bipolaires, la dépression psychotique, la démence, l'autisme, les troubles développementaux, etc. (6-8).

Les données de la littérature concernant les troubles métaboliques dans des troubles mentaux autres que la schizophrénie sont limitées. Cependant, certains patients, entre autres ceux ayant des troubles de l'humeur, présentent également un risque accru (8-9-28).

Dès lors, nous devons prendre des mesures de prudence; ces patients seront détectés et suivis tout comme les patients psychotiques. Les patients recevant des antipsychotiques en tant que stabilisateurs de l'humeur et connus pour induire un gain pondéral seront suivis avec précaution. Ces patients et leur famille, ainsi que les soignants, seront avertis et conseillés quant à une possible complication métabolique. Si celle-ci survient, un changement vers une médication possédant un risque moindre de troubles métaboliques sera pris en compte.

\section{INFORMATION DU PATIENT À PROPOS DU TROUBLE MÉTABOLIQUE INDUIT PAR UN ANTIPSYCHOTIQUE}

Lors de l'initiation d'un antipsychotique, le besoin d'un antipsychotique ainsi qu'une thérapie de maintenance seront évoqués. Le risque de la non-observation et les avantages des antipsychotiques atypiques seront discutés. Le patient sera informé quant aux risques métaboliques de sa pathologie et du risque associé induit par les antipsychotiques. L'information inclura le risque de diabète et d'hyperglycémie, de gain de poids, de dyslipémie. Le praticien prendra des mesures préventives telles qu'un changement de style de vie et un régime.

Du fait qu'un diabète de type I ne peut être prévu, l'éducation des patients à ces risques est recommandée. De façon plus spécifique, le patient et les membres de sa famille ainsi que les donneurs de soins devront être infor- més des signes aigus de décompensation acidocéto-diabétique et des risques vitaux, quoique peu fréquents, qu'elle entraîne (6).

Une acido-acétose diabétique inclut un début d'apparition rapide de polyurie et de polydipsie, une perte de poids, des nausées et des vomissements, une déshydratation, une respiration rapide et une perte de vigilance pouvant aller jusqu'au coma.

\section{CONCLUSION}

Les SGA sont devenus la pierre angulaire du traitement des troubles psychotiques de la schizophrénie. C'est une évidence croissante que certains SGA sont associés à un risque plus élevé de troubles métaboliques.

Avant d'entreprendre un traitement par SGA, tous les facteurs de risques métaboliques doivent être considérés. Lors du choix du SGA, il y a lieu de soigneusement soupeser, à efficacité égale, à la fois le risque d'induction d'un trouble métabolique et les risques de facteurs métaboliques intrinsèques au patient. II faut privilégier le SGA aux facteurs de risques métaboliques moindres.

Les stades précoces du diabète, de l'obésité, des dyslipémies doivent être décelés durant les premiers mois du traitement et si nécessaire traités. Un arrêt du SGA impliqué ainsi qu'un switch vers un SGA à risque moindre fait partie de nos recommandations.

Enfin, le psychiatre doit informer et créer une collaboration avec son patient, sa famille et les autres soignants tels que les généralistes et/ou l'interniste.

Pour effectuer le suivi clinique, nous éditerons une « feuille de route » reprenant sous forme de graphique les différents paramètres ainsi que la chronologie à prendre en compte. Nous nous proposons de les diffuser largement.

Nous nous distinguons des deux autres consensus anglo-américains par la recommandation d'une fréquence plus grande des contrôles lipidiques, par la préférence de l'hyperglycémie provoquée versus la glycémie à jeun et par l'établissement d'une hiérarchie de risque d'induction de troubles métaboliques selon le type de SGA utilisé.

\section{ANNEXE 1}

\section{Membres du consensus}

Co-Chairmans : A.R. De Nayer, J. Peuskens.

Participants : C. Bervoets (PZ Onze Lieve Vrouw, Brugge) ; M. De Hert (UC St-Jozef, Kortenberg) ; A.R. De Nayer (CH Ste-Thérèse, Gilly) ; J.M. Devoitille (CH Psychiatrique de Liège - Le Petit Bourgogne) ; J.M. Domken (CHP du « Chêne aux Haies ", Mons); J. Hulselmans (PZ Stuivenberg, Antwerpen); D. Lecompte (CHU-Brugman, Bruxelles) ; F. Lotstra (CHU Erasme, Bruxelles); F. Mathot (CH Psychiatrique de Liège - Le Petit Bourgogne) ; C. Mertens (PZ St-Camillus, Gent) ; J. Peuskens (UC St-Jozef, Kortenberg) ; J. Reyntens (Clinical Pharmacist Psychiatrisch Centrum Sint-Jan Eeklo) ; A. Scheen (Division of Diabetes, Nutrition and Metabolic Disorders, Department of Medicine, CHU Sart Tilman, Liège) ; A. Seghers (CHU St-Luc, Bruxelles) ; E. Stillemans (CH Jean Titeca, Bruxelles) ; D. Van Eyck (UC St-Jozef, Kortenberg) ; L. Van Gaal (Deptartment of Diabetology, Metabolism and Nutrition, Antwerp) ; F. Vandendriessche (PC Sint-Norbertus, Duffel). 


\section{Références}

1. AMERICAN DIABETES ASSOCIATION. Standards of medical care for patients with diabetes mellitus. Diabetes Care 2002 ; 26 (Suppl 1) : 33-50.

2. CARO JJ, WARD A, LEVINTON C et al. The risk of diabetes during olanzapine use compared with risperidone use : a retrospective database analysis. J Clin Psychiatry 2002 ; 63 : 1135-9.

3. CITROME LL, JAFFE AB. Relationship of atypical antipsychotics with development of diabetes mellitus. Ann Pharmacother 2003 ; 37 : 1849-57.

4. CLARK C, BURGE MR. Diabetes mellitus associated with atypical antipsychotic medications. Diabetes Technol Ther $2003 ; 5: 669-83$.

5. COHN T, WOLEVER T, ZIPURSKY R et al. Screening for diabetes and impaired glucose tolerance in patients on antipsychotic medication. J Neuropsychopharmacol 2002 ; 5 (Suppl I) : 168.

6. CONSENSUS DEVELOPMENT CONFERENCE ON ANTIPSYCHOTIC DRUGS AND OBESITY AND DIABETES. Diabetes Care $2004 ; 27: 596-601$.

7. DE HERT M, VAN EYCK D, PEUSKENS $\mathrm{H}$ et al. Prevalence of impaired glucose tolerance in schizophrenic patients [abstract]. Annual Meeting of the American Psychiatric Society, 2004.

8. GIANFRANCESCO FD, GROGG AL, MAHMOUD RA et al. Differential effects of risperidone, olanzapine, clozapine and conventional antipsychotics on type 2 diabetes : findings from a large health plan database. J Clin Psychiatry 2002 ; 63 : 920-30.

9. GIANFRANCESCO F, GROGG A, MAHMOUD R et al. Differential effects of antipsychotic agents on the risk of development of type 2 diabetes mellitus in patients with mood disorders. Clin Ther 2003 ; $25: 1150-71$.

10. GOUGH S, PELEVER R. Diabetes and its prevention : pragmatic solutions for people with schizophrenia. Br J Psychiatry 2004 ; 184 : S106-S111.

11. GUERY C. Schizophrénie, diabète et antipsychotique. Encephale $2004 ; 30$ (4) : 382-91.

12. HAUPT DW, NEWCOMER JW. Hyperglycemia and antipsychotic medications. J Clin Psychiatry 2001; 62 (Suppl 27) : 15-26 ; discussion : 40-1.

13. HEDENMALM K, HAGG S, STAHL M et al. Glucose intolerance with atypical antipsychotics. Drug Saf $2002 ; 25: 1107-16$.

14. HENDERSON DC. Atypical antipsychotic-induced diabetes mellitus : how strong is the evidence ? CNS Drugs $2002 ; 16: 77-89$.

15. HOWES OD, BHATNAGAR, FP GAUGHRAN et al. The effect of clozapine on factors controlling glucose homeostasis. Clin Neuropsychopharmacol S67 ; poster APA 2005.

16. KNOWLER WC, BARRETT-CONNOR E, FOWLER SE et al. Reduction in the incidence of type 2 diabetes with lifestyle intervention or metformin. N Engl J Med 2002 ; 346 : 393-403.
17. KOLLER EA, CROSS JT, DORAISWAMY PM et al. Risperidoneassociated diabetes mellitus : a pharmacovigilance study. Pharmacotherapy $2003 ; 23: 735-44$.

18. KOLLER EA, DORAISWAMY PM. Olanzapine-associated diabetes mellitus. Pharmacotherapy $2002 ; 22: 841-52$.

19. KOLLER E, SCHNEIDER B, BENNETT K et al. Clozapine-associated diabetes. Am J Med $2001 ; 111: 716-23$.

20. KOLLER EA, WEBER J, DORAISWAMY PM et al. A survey of reports of quetiapine-associated hyperglycemia and diabetes mellitus. J Clin Psychiatry $2004 ; 65: 857-63$.

21. KORO CE, FEDDER DO, L'ITALIEN GJ et al. Assessment of independent effect of olanzapine and risperidone on risk of diabetes among patients with schizophrenia : population based nested casecontrol study. Br Med J $2002 ; 325: 243$.

22. MARDER SR, ESSOCK SM, MILLER AL et al. Physical health monitoring of patients with schizophrenia. Am J Psychiatry 2004 ; 161 : 1334-49.

23. MELKERSSON K, DAHL ML. Adverse metabolic effects associated with atypical antipsychotics : literature review and clinical implications. Drugs $2004 ; 64: 701-23$.

24. MEYER JM, KORO CE. The effects of antipsychotic therapy on serum lipids : a comprehensive review. Schizophr Res 2004 ; 70 : 1-17.

25. MUKHERJEE S, DECINA P, BOCOLA V et al. Diabetes mellitus in schizophrenic patients. Compr Psychiatry $1996 ; 37: 68-73$.

26. NASRALLAH HA, KORN ML. Metabolic disorders in schizophrenia : relationship to atypical antipsychotic treatment. Posted 07/28/2004 : Medscape Psychiatry and Mental Health Schizophrenia Expert Column, 2004.

27. PALMER JP, HIRSCH IB. What's in a name : latent auto-immune diabetes of adults, type 1.5, adult-onset, and type 1 diabetes. Diabet Care $2003 ; 26: 536-8$.

28. REGENOLD WT, THAPAR RK, MARANO $\mathrm{C}$ et al. Increased prevalence of type 2 diabetes mellitus among psychiatric inpatients with bipolar I affective and schizoaffective disorders independent of psychotropic drug use. J Affect Disord 2002 ; 70 : 19-26.

29. ROSACK J. FDA to require diabetes warning on antipsychotics. Psychiatr News $2003 ; 38: 1-a-27$.

30. «SCHIZOPHRENIA AND DIABETES 2003 ». Expert Consensus Meeting, Dublin, 3-4 October 2003 : consensus summary. Br J Psychiatry $2004 ; 184:$ S112-4.

31. SERNYAK MJ, LESLIE DL, ALARCON RD et al. Association of diabetes mellitus with use of atypical neuroleptics in the treatment of schizophrenia. Am J Psychiatry 2002 ; 159 : 561-6.

32. SUBRAMANIAM M, CHONG SA, PEK E. Diabetes mellitus and impaired glucose tolerance in patients with schizophrenia. Can J Psychiatry $2003 ; 48: 345-7$. 